\title{
Schrödinger-type identity for Schrödinger free boundary problems
}

\author{
Xingjian Zhang ${ }^{1}$, Duo Liu', Zining Yan', Guodong Zhao ${ }^{1 *}$ and Ye Yuan'
}

"Correspondence:
zgudo@foxmail.com
'Department of Computer Science
and Technology, Harbin
Engineering University, Harbin,
China

"Correspondence: zgudo@foxmail.com and Technology, Harbin Enina

\begin{abstract}
Our aim in this paper is to develop a Schrödinger-type identity for a Schrödinger free boundary problem in $\mathbb{R}^{n}$. As an application, we establish necessary and sufficient conditions for the product of some distributional functions to satisfy the Schrödinger-type identity. As a consequence, our results significantly improve and generalize previous work.
\end{abstract}

Keywords: Schrödinger-type identity; Schrödinger transform; Distribution

\section{Introduction and main results}

Schrödinger-type identities have been studied extensively in the literature (see $[1,12,13$, $18]$ for the Schrödinger equation, $[5,14]$ for Schrödinger systems).

In recent years, many exciting phenomena were found by careful experiments on light waves propagating in nonlinear periodic lattices. These phenomena are governed by the following Schrödinger equation:

$$
\operatorname{Sch}_{\alpha}(u)=(-\Delta)^{\alpha} u+V(x) u-h(x, u)=0
$$

in $\mathbb{R}^{n}$, where $n \geq 2, \alpha \in(0,1),(-\Delta)^{\alpha}$ stands for the fractional Laplacian, $V$ is a positive continuous potential, $h \in C\left(\mathbb{R}^{2} \times \mathbb{R}, \mathbb{R}\right)$. The fractional Laplacian $(-\Delta)^{\alpha}$ with $\alpha \in(0,1)$ of a function $\iota \in \mathcal{S}$ is defined by

$$
\mathcal{G}\left(\left((-\Delta)^{\alpha}\right) \iota\right)(\xi)=|\xi|^{2 \alpha} \mathcal{G}(\iota)(\xi), \quad \forall \alpha \in(0,1)
$$

where $\mathcal{S}$ denotes the Schwartz space of rapidly decreasing $C^{\infty}$ functions in $\mathbb{R}^{n}$ and

$$
\mathcal{F}(\iota)(\xi)=\frac{1}{(2 \pi)^{n / 2}} \int_{\mathbb{R}^{N}} e^{-2 \pi i \xi \cdot x} \iota(x) d x .
$$

The Schrödinger transform $\mathrm{Sch}_{\alpha}$ is defined as the following singular integral:

$$
\left(\operatorname{Sch}_{\alpha}(f)\right)(x):=p \cdot v \cdot \frac{1}{\pi} \int_{\mathbb{R}} \frac{f(y)}{x-y} d y=\lim _{\epsilon \rightarrow 0} \int_{|y-x| \geq \epsilon} \frac{f(y)}{x-y} d y,
$$

where $x \in \mathbb{R}$.

(c) The Author(s) 2018. This article is distributed under the terms of the Creative Commons Attribution 4.0 International License (http://creativecommons.org/licenses/by/4.0/), which permits unrestricted use, distribution, and reproduction in any medium, provided you give appropriate credit to the original author(s) and the source, provide a link to the Creative Commons license, and indicate if changes were made. 
The Schrödinger-type identity for Schrödinger free boundary problems

$$
\operatorname{Sch}_{\alpha}(f g)=\mathrm{fSch}_{\alpha}(g)
$$

was first studied in [2-4, 6]. It was proved that the above identity holds if $h, g \in L^{2}(\mathbb{R})$ satisfy supp $\hat{f} \subseteq \mathbb{R}_{+}\left(\mathbb{R}_{+}=[0, \infty)\right)$ and supp $\hat{g} \subseteq \mathbb{R}_{+}$in [20]. In 2015, Wan also obtained more general sufficient conditions by weakening the above condition in [19]. Recently, Lv and Ulker and Huang established the first necessary and sufficient condition in the time domain and a parallel result in the frequency domain for the Poisson inequality in [10, 14].

It is natural that there have been attempts to define the complex signal and prove the Schrödinger-type identity in the multidimensional case.

Definition 1.1 The partial Schrödinger transform $\operatorname{Sch}_{\alpha j}$ of $f$ is given by

$$
\left(\operatorname{Sch}_{\alpha j} f\right)(x):=p \cdot v \cdot \frac{1}{\pi} \int_{\mathbb{R}} \frac{f(y)}{x_{j}-y_{j}} d y_{j},
$$

where $f \in L^{p}\left(\mathbb{R}_{n}\right)$ and $1 \leq p<\infty$.

The total Schrödinger transform $\operatorname{Sch}_{\alpha}$ of $f$ is defined as follows:

$$
\begin{aligned}
\left(\operatorname{Sch}_{\alpha}(f)\right)(x) & :=p \cdot v \cdot \frac{1}{\pi^{n}} \int_{\mathbb{R}^{n}} \frac{f(y)}{\prod_{j=1}^{n}\left(x_{j}-y_{j}\right)} d y \\
& =\lim _{\max \epsilon_{j} \rightarrow 0} \int_{\left|y_{j}-x_{j}\right| \geq \epsilon_{j}>0, j=1,2, \ldots, n} \frac{f(y)}{\prod_{j=1}^{n}\left(x_{j}-y_{j}\right)} d y,
\end{aligned}
$$

where $f \in L^{p}\left(\mathbb{R}_{n}\right)$ and $1 \leq p<\infty$. The property

$$
\left\|\operatorname{Sch}_{\alpha}(f)\right\|_{L^{p}\left(\mathbb{R}^{n)}\right.} \leq C_{p}^{n}\|f\|_{L^{p}\left(\mathbb{R}^{n}\right)}
$$

was proved in [8]. The iterative nature of it in $L^{p}\left(\mathbb{R}^{n}\right)$ was shown in [16], where $p>1$. It was shown that

$$
\operatorname{Sch}_{\alpha}=\prod_{j=1}^{n} \operatorname{Sch}_{\alpha j}
$$

The operations $\operatorname{Sch}_{\alpha i}$ and $\operatorname{Sch}_{\alpha j}$ commute with each other, where $i, j=1,2, \ldots, n$.

Now we define the Schrödingerean Fourier transform $\hat{f}$ of $f$ (see [17]) by

$$
\hat{f}(x)=\int_{\mathbb{R}^{n}} f(t) e^{-i x . t} d t
$$

where $x \in \mathbb{R}^{n}$ and $f \in L^{1}\left(\mathbb{R}^{n}\right)$.

Set

$$
D_{+}=\left\{x: x \in \mathbb{R}^{n}, \operatorname{sgn}(-x)=\prod_{j=1}^{n} \operatorname{sgn}\left(-x_{j}\right)=1\right\},
$$




$$
D_{-}=\left\{x: x \in \mathbb{R}^{n}, \operatorname{sgn}(-x)=\prod_{j=1}^{n} \operatorname{sgn}\left(-x_{j}\right)=-1\right\},
$$

and

$$
D_{0}=\left\{x: x \in \mathbb{R}^{n}, \operatorname{sgn}(-x)=\prod_{j=1}^{n} \operatorname{sgn}\left(-x_{j}\right)=0\right\} .
$$

We denote by $\mathcal{D}_{D_{+}}\left(\mathbb{R}^{n}\right), \mathcal{D}_{D_{-}}\left(\mathbb{R}^{n}\right)$ and $\mathcal{D}_{D_{0}}\left(\mathbb{R}^{n}\right)$ the set of functions in $\mathcal{D}\left(\mathbb{R}^{n}\right)$ that are supported on $D_{+}, D_{-}$, and $D_{0}$, respectively.

The Schwartz class $\mathcal{S}\left(\mathbb{R}^{n}\right)$ consists of all functions $\varphi$ on $\mathbb{R}^{n}$ such that

$$
\sup _{x \in \mathbb{R}^{n}}\left|x^{\alpha} D^{\beta} \varphi(x)\right|<\infty
$$

where $\alpha, \beta \in \mathbb{Z}_{+}^{n}$.

The Schrödingerean Fourier transform $\hat{\varphi}$ is a linear homeomorphism from $S\left(\mathbb{R}^{n}\right)$ onto itself. Meanwhile, the following identity holds:

$\left(\operatorname{Sch}_{\alpha} \varphi\right)^{\wedge}(x)=(-i) \operatorname{sgn}(x) \hat{\varphi}$,

where $\varphi \in \mathcal{S}\left(\mathbb{R}^{n}\right)$.

The Schrödingerean Fourier transform $F: \mathbb{S}^{\prime}\left(\mathbb{R}^{n}\right) \rightarrow \mathbb{S}^{\prime}\left(\mathbb{R}^{n}\right)$ is defined for any $\varphi \in \mathcal{S}\left(\mathbb{R}^{n}\right)$ as follows:

$$
\langle\hat{\varrho}, \varphi\rangle=\langle\varrho, \hat{\varphi}\rangle,
$$

which is a linear isomorphism from $\mathbb{S}^{\prime}\left(\mathbb{R}^{n}\right)$ onto itself. For the detailed properties of $\mathbb{S}\left(\mathbb{R}^{n}\right)$ and $\mathbb{S}^{\prime}\left(\mathbb{R}^{n}\right)$, see $[3,7,15]$.

For $\varrho \in \mathcal{S}^{\prime}\left(\mathbb{R}^{n}\right), \lambda \in \mathcal{S}\left(\mathbb{R}^{n}\right)$, it is easy to check that

$$
\langle\tilde{\varrho}, \lambda\rangle=\langle\tilde{\varrho}, \hat{\lambda}\rangle=\langle\varrho, \tilde{\tilde{\lambda}}\rangle=\langle\hat{\varrho}, \lambda\rangle=\langle\varrho, \hat{\lambda}\rangle
$$

for any $\lambda \in \mathcal{S}\left(\mathbb{R}^{n}\right)$, where

$$
\tilde{\lambda}(x)=\lambda(-x),
$$

$\tilde{\varrho}$ is the inverse Schrödingerean Fourier transform defined as follows:

$$
\langle\breve{\varrho}, \lambda\rangle=\langle\varrho, \tilde{\lambda}\rangle .
$$

Therefore in the distributional sense, we obtain

$$
\tilde{\varrho}=\hat{\varrho} .
$$

Following the definition in [4], a function $\lambda$ belongs to the space $\mathcal{D}_{L^{p}}\left(\mathbb{R}^{n}\right), 1 \leq p<\infty$ if and only if 
(1) $\lambda \in C^{\infty}\left(\mathbb{R}^{n}\right)$;

(2) $D^{k} \lambda \in L^{p}\left(\mathbb{R}^{n}\right), k=1,2, \ldots$, where $C^{\infty}\left(\mathbb{R}^{n}\right)$ consists of infinitely differentiable functions,

$$
D^{k} \lambda(x)=\frac{\partial^{|k|}}{\partial x_{1}^{k_{1}} \cdots \partial x_{n}^{k_{n}}} \lambda(x)
$$

In the sequel, we denote by $\mathcal{D}_{L^{p}}^{\prime}\left(\mathbb{R}^{n}\right)$ the dual of the corresponding spaces $\mathcal{D}_{L^{p^{p}}}\left(\mathbb{R}^{n}\right)$, where

$$
\frac{1}{p}+\frac{1}{p^{\prime}}=1
$$

As a consequence, we have

$$
\mathcal{D}\left(\mathbb{R}^{n}\right) \subseteq \mathcal{S}\left(\mathbb{R}^{n}\right) \subseteq \mathcal{D}_{L^{p}}\left(\mathbb{R}^{n}\right) \subseteq L^{p}\left(\mathbb{R}^{n}\right)
$$

and

$$
L^{p}\left(\mathbb{R}^{n}\right) \subseteq \mathcal{D}_{L^{p}}^{\prime}\left(\mathbb{R}^{n}\right) \subseteq \mathcal{S}^{\prime}\left(\mathbb{R}^{n}\right) \subseteq \mathcal{D}^{\prime}\left(\mathbb{R}^{n}\right)
$$

Definition 1.2 Let $f \in \mathcal{D}_{L^{p}}^{\prime}\left(\mathbb{R}^{n}\right)$, where $1<p<\infty$. Then the Schrödinger transform of $f$ is defined as follows:

$$
\left\langle\operatorname{Sch}_{\alpha}(f), \lambda\right\rangle=\left\langle f,(-1)^{n} \operatorname{Sch}_{\alpha} \lambda\right\rangle,
$$

where $\lambda \in \mathcal{D}_{L^{p^{\prime}}}\left(\mathbb{R}^{n}\right)$.

In [10], Huang proved that the total Schrödinger transform is a linear homeomorphism from $\mathcal{D}_{L^{p}}\left(\mathbb{R}^{n}\right)$ onto itself, and that, if $h \in \mathcal{D}_{L^{p}}^{\prime}\left(\mathbb{R}^{n}\right)(1<p<\infty)$, then $\mathfrak{P} \Im h \in \mathcal{D}_{L^{p}}^{\prime}\left(\mathbb{R}^{n}\right)$ and the Schrödinger transform $\mathrm{H}$ defined above is a linear isomorphism from $\mathcal{D}_{L^{p}}^{\prime}\left(\mathbb{R}^{n}\right)$ onto itself.

Note that, if $\varrho \in L^{p}\left(\mathbb{R}^{n}\right)(1<p<\infty)$, then we have

$$
\begin{aligned}
\left\langle(H \varrho)^{\wedge}, \lambda\right\rangle & =\langle H \varrho, \hat{\lambda}\rangle \\
& =(-1)^{n}\langle\varrho, H \hat{\lambda}\rangle \\
& =(-1)^{n}\left\langle\check{\varrho},(H \hat{\lambda})^{\wedge}\right\rangle \\
& =(-1)^{n}\left\langle\check{\varrho},(-i)^{n} \operatorname{sgn}(\cdot) \hat{\hat{\lambda}}\right\rangle \\
& =\left\langle\check{\varrho},(i)^{n} \operatorname{sgn}(\cdot) \tilde{\lambda}\right\rangle \\
& =\left\langle\tilde{\varrho},(i)^{n} \operatorname{sgn}(\cdot) \lambda\right\rangle \\
& =\left\langle(-i)^{n} \operatorname{sgn}(\cdot) \hat{\varrho}, \lambda\right\rangle,
\end{aligned}
$$

where $\lambda \in \mathcal{S}\left(\mathbb{R}^{n}\right)$.

Therefore in the distributional sense

$$
(H \varrho)^{\wedge}(x)=(-i)^{n} \operatorname{sgn}(\cdot) \hat{\varrho}(x)
$$


Define

$$
t \Omega=\{t x: x \in \Omega\}
$$

where $t$ is a nonzero real number and $\Omega$ is a nonempty subset of $\mathbb{R}$. Hence we have

$$
\operatorname{supp}\left(u\left(\frac{x}{t}\right)\right)=t \operatorname{supp}(u)
$$

for any nonzero real number $t$.

For a subset $A \subseteq \mathbb{R}$, define

$$
A \Omega=\bigcup_{t \in A} t \Omega
$$

\section{Schrödinger-type identity for $L^{p}\left(\mathbb{R}^{n}\right)$ functions}

This part is motivated by the need of defining multidimensional complex signals. We define the complex signal of $f \in L^{p}\left(\mathbb{R}^{n}\right)$ through the total Schrödinger transform $\operatorname{Sch}_{\alpha}$ as $f+i \operatorname{Sch}_{\alpha}(f)$.

In this section we investigate the multidimensional Schrödinger-type identity $\operatorname{Sch}_{\alpha}(f g)=$ $\mathrm{fSch}_{\alpha}(g)$ for $f \in \mathcal{S}\left(\mathbb{R}^{n}\right)$ and $g \in L^{p}\left(\mathbb{R}^{n}\right)$, where $1<p \leq 2$. In particular, several necessary and sufficient conditions are obtained.

Theorem 2.1 Suppose that $f \in \mathcal{S}\left(\mathbb{R}^{n}\right) ; g \in L^{p}\left(\mathbb{R}^{n}\right)(1<p \leq 2)$, then the Schrödinger transform of the function $f g$ satisfies the Schrödinger-type identity $\operatorname{Sch}_{\alpha}(f g)=\mathrm{fSch}_{\alpha}(g)$ if and only if

$$
\int_{\mathbb{R}^{n}}(\operatorname{sgn}(x)-\operatorname{sgn}(t)) \hat{f}(x-t) \hat{g}(t) \mathrm{d} t=0 .
$$

Proof According to [10], we use the following equalities:

$$
\begin{aligned}
& \mathrm{d} \bar{x}=\varepsilon^{2} \mathrm{~d} x, \\
& \mathrm{~d} \bar{\Gamma}=\varepsilon \mathrm{d} \Gamma \quad \text { on } \mathcal{S}\left(\mathbb{R}^{n}\right), \\
& \mathrm{d} \bar{\Gamma}=\varepsilon^{2} \mathrm{~d} \Gamma \quad \text { on } L^{p}\left(\mathbb{R}^{n}\right) .
\end{aligned}
$$

So

$$
\begin{aligned}
& \int_{0}^{T} \int_{\mathbb{R}^{n}}\left(f_{\varepsilon} \partial_{t} \lambda+f_{\varepsilon} g_{\varepsilon} \cdot \nabla_{\varepsilon} \lambda\right) \mathrm{d} x \mathrm{~d} t=0, \\
& \int_{0}^{T} \int_{\mathbb{R}^{n}}\left[f_{\varepsilon} g_{\varepsilon} \cdot \partial_{t} \varrho+f_{\varepsilon} g_{\varepsilon} \otimes g_{\varepsilon}: \omega_{\varepsilon}(\varrho)+f_{\varepsilon} \operatorname{div}_{\varepsilon} \varrho\right] \mathrm{d} x \mathrm{~d} t \\
& \quad \int_{0}^{T} \int_{\mathbb{R}^{n}}\left[P\left(\left|\omega_{\varepsilon}\left(g_{\varepsilon}\right)\right|\right) \omega_{\varepsilon}\left(g_{\varepsilon}\right): \omega_{\varepsilon}(\varrho)-f_{\varepsilon} \cdot \varrho\right] \mathrm{d} x \mathrm{~d} t \\
& \quad+\frac{h(\varepsilon)}{\varepsilon} \int_{0}^{T} \int_{\mathcal{S}\left(\mathbb{R}^{n}\right)} g_{\varepsilon} \cdot \varrho \mathrm{d} \Gamma \mathrm{d} t+q \int_{0}^{T} \int_{L^{p}\left(\mathbb{R}^{n}\right)} g_{\varepsilon} \cdot \varrho \mathrm{d} \Gamma \mathrm{d} t,
\end{aligned}
$$


for any $\varrho \in C_{0}^{\infty}\left(0, T ;\left[C^{\infty}(\bar{\Omega})\right]^{3}\right)$, which leads to

$$
\int_{0}^{T} \int_{\Omega} b\left(f_{\varepsilon}\right) \partial_{t} \lambda+b\left(f_{\varepsilon}\right) g_{\varepsilon} \cdot \nabla_{\varepsilon} \lambda+\left[\left(b\left(f_{\varepsilon}\right)-f_{\varepsilon} b^{\prime}\left(f_{\varepsilon}\right)\right) \operatorname{div}_{\varepsilon} g_{\varepsilon}\right] \lambda \mathrm{d} x \mathrm{~d} t=0
$$

Note that (see [19])

$$
\begin{aligned}
\int_{\Omega_{\varepsilon}} & \left(\bar{\rho}_{\varepsilon}(t) \frac{\left|\bar{g}_{\varepsilon}(t)\right|^{2}}{2}+\bar{\rho}_{\varepsilon}(t) \ln \left(\bar{\rho}_{\varepsilon}(t)\right)\right) \mathrm{d} \bar{x} \\
& +\int_{0}^{t} \int_{\Omega_{\varepsilon}} P\left(\left|\bar{D} \bar{g}_{\varepsilon}\right|\right) \bar{D} \bar{g}_{\varepsilon}: \bar{D}_{\bar{g}} \mathrm{~d} \bar{x} \mathrm{~d} s+h(\varepsilon) \int_{0}^{t} \int_{\Gamma_{1, \varepsilon}}\left|\bar{g}_{\varepsilon}\right|^{2} \mathrm{~d} \bar{\Gamma} \mathrm{d} s \\
& +q \int_{0}^{t} \int_{\Gamma_{2, \varepsilon}}\left|\bar{g}_{\varepsilon}\right|^{2} \mathrm{~d} \bar{\Gamma} \mathrm{d} s \\
= & \int_{0}^{t} \int_{\Omega_{\varepsilon}} \bar{\rho}_{\varepsilon} \overline{\mathbf{f}}_{\varepsilon} \cdot \bar{g}_{\varepsilon} \mathrm{d} \bar{x} \mathrm{~d} s+\int_{\Omega_{\varepsilon}}\left(\frac{\left|\left(\bar{\rho}_{\varepsilon} \bar{g}_{\varepsilon}\right)_{0}\right|^{2}}{2 \bar{\rho}_{0, \varepsilon}}+\bar{\rho}_{0, \varepsilon} \ln \left(\bar{\rho}_{0, \varepsilon}\right)\right) \mathrm{d} \bar{x} .
\end{aligned}
$$

For any $t \in\langle 0, T\rangle$, this yields

$$
\begin{aligned}
& \int_{\Omega}(\left.f_{\varepsilon}(t) \frac{\left|g_{\varepsilon}(t)\right|^{2}}{2}+f_{\varepsilon}(t) \ln \left(f_{\varepsilon}(t)\right)\right) \mathrm{d} x \\
&+\int_{0}^{t} \int_{\Omega} P\left(\left|\omega_{\varepsilon}\left(g_{\varepsilon}\right)\right|\right)\left|\omega_{\varepsilon}\left(g_{\varepsilon}\right)\right|^{2} \mathrm{~d} x \mathrm{~d} s \\
&+\frac{h(\varepsilon)}{\varepsilon} \int_{0}^{t} \int_{\mathcal{S}\left(\mathbb{R}^{n}\right)}\left|g_{\varepsilon}\right|^{2} \mathrm{~d} \Gamma \mathrm{d} s+q \int_{0}^{t} \int_{L^{p}\left(\mathbb{R}^{n}\right)}\left|g_{\varepsilon}\right|^{2} \mathrm{~d} \Gamma \mathrm{d} s \\
&= \int_{0}^{t} \int_{\Omega} f_{\varepsilon} \mathbf{g}_{\varepsilon} \cdot \mathbf{v}_{\varepsilon} \mathrm{d} x \mathrm{~d} s+\int_{\Omega}\left(\frac{\left|\left(f_{\varepsilon} g_{\varepsilon}\right)_{0}\right|^{2}}{2 f_{0, \varepsilon}}+f_{0, \varepsilon} \ln \left(f_{0, \varepsilon}\right)\right) \mathrm{d} x \\
&\left\|\sqrt{g_{\varepsilon}} \bar{\partial} \alpha\right\|_{\lambda}^{2}+\left\|\sqrt{g_{\varepsilon}} \bar{\partial}_{\lambda}^{*} \alpha\right\|_{\lambda}^{2} \\
&=\sum_{|L|=p-1}^{\prime} \sum_{j, k=1}^{n} \int_{b \Omega} g \frac{\partial^{2} \rho}{\partial z_{j} \partial \bar{z}_{k}} \alpha_{j L} \bar{\alpha}_{k L} e^{-\lambda} d S \\
& \quad+\sum_{|K|=p}^{\prime} \sum_{k=1}^{n} \int_{\Omega} g\left|\frac{\partial \alpha_{K}}{\partial \bar{z}_{k}}\right|^{2} e^{-\lambda} d V \\
& \quad+\sum_{|L|=p-1}^{\prime} \sum_{j, k=1}^{n} \int_{\Omega}\left(g \frac{\partial^{2} \lambda}{\partial z_{j} \partial \bar{z}_{k}}-\frac{\partial^{2} g}{\partial z_{j} \partial \bar{z}_{k}}\right) \alpha_{j L} \bar{\alpha}_{k L} e^{-\lambda} d V \\
& \quad+2 \operatorname{Re}\left|\sum_{\mid L L=p-1}^{\prime} \sum_{j=1}^{n} \alpha_{j L} \frac{\partial g}{\partial z_{j}} d \bar{z}_{L}, \bar{\partial}_{\lambda}^{*} \alpha\right\rangle_{\lambda}
\end{aligned}
$$

and

$$
\begin{aligned}
& 2 \operatorname{Re}\left\langle\sum_{|L|=p-1}{ }^{\prime} \sum_{j=1}^{n} \alpha_{j L} \frac{\partial g}{\partial z_{j}} d \bar{z}_{L}, \bar{\partial}_{\lambda}^{*} \alpha\right\rangle_{\lambda} \\
& \quad \leq 2\left|\left\langle\sum_{|L|=p-1}{ }^{\prime} \frac{1}{\sqrt{g_{\varepsilon}}} e^{-\lambda / 2} \sum_{j=1}^{n} \frac{\partial g}{\partial z_{j}} \alpha_{j L} d \bar{z}_{j}, \sqrt{g_{\varepsilon}} e^{-\lambda / 2} \bar{\partial}_{\lambda}^{*} \alpha\right\rangle\right|
\end{aligned}
$$




$$
\begin{aligned}
& \leq 2\left\|\sum_{|L|=p-1}{ }^{\prime} \frac{1}{\sqrt{g_{\varepsilon}}} \sum_{j=1}^{n} \frac{\partial g}{\partial z_{j}} \alpha_{j L} d \bar{z}_{j}\right\|_{\lambda}\left\|\sqrt{g_{\varepsilon}} \bar{\partial}_{\lambda}^{*} \alpha\right\|_{\lambda} \\
& \leq \sum_{|L|=p-1}{ }^{\prime} \zeta\left\|\frac{1}{\sqrt{g_{\varepsilon}}} \sum_{j=1}^{n} \frac{\partial g}{\partial z_{j}} \alpha_{j L}\right\|_{\lambda}^{2}+\frac{1}{\zeta}\left\|\sqrt{g_{\varepsilon}} \bar{\partial}_{\lambda}^{*} \alpha\right\|_{\lambda}^{2}
\end{aligned}
$$

for any $t \in\langle 0, T\rangle$, where

$$
g_{\varepsilon}=\left(f_{1, \varepsilon}, \varepsilon^{-1} f_{2, \varepsilon}, \varepsilon^{-1} f_{3, \varepsilon}\right), \quad v_{\varepsilon}=\left(u_{1, \varepsilon}, \varepsilon u_{2, \varepsilon}, \varepsilon u_{3, \varepsilon}\right) .
$$

Since the Schrödingerean Fourier transform is injective from $\mathcal{S}^{\prime}$ into itself, $f g, \operatorname{Sch}_{\alpha}(f) g$, $\mathrm{fSch}_{\alpha}(g) \in L^{p}\left(\mathbb{R}^{n}\right)$, we have

$$
\left(\operatorname{Sch}_{\alpha}(f g)\right)^{\wedge}=\left(\operatorname{fSch}_{\alpha}(g)\right)^{\wedge}
$$

which is equivalent to

$$
(-1)^{n} \operatorname{sgn}(x) \int_{\mathbb{R}^{n}} \hat{f}(x-t) \hat{g_{\varepsilon}}(t) d t=\int_{\mathbb{R}^{n}}(-1)^{n} \operatorname{sgn}(t) \hat{f}(x-t) \hat{g_{\varepsilon}}(t) d t,
$$

where

$$
\operatorname{sgn}(x)=\prod_{j}^{n}=1 \operatorname{sgn}\left(x_{j}\right), \quad x=\left(x_{1}, x_{2}, \ldots, x_{n}\right) .
$$

So

$$
\int_{\mathbb{R}^{n}}(\operatorname{sgn}(x)-\operatorname{sgn}(t)) \hat{f}(x-t) \hat{g_{\varepsilon}}(t) d t=0 .
$$

Let $a_{j}$ and $b_{j}$ denote nonnegative real numbers in the rest of the paper, where $j=$ $1,2, \ldots, n$.

Corollary 2.1 Let $f \in \mathcal{S}\left(\mathbb{R}^{n}\right)$ and $g \in L^{p}\left(\mathbb{R}^{n}\right)$, where $1<p \leq 2$. If

$$
\operatorname{supp} \hat{f} \subseteq \prod_{j=1}^{n}\left[-a_{j}, b_{j}\right], \quad \operatorname{supp} \hat{g_{\varepsilon}} \subseteq \prod_{j=1}^{n} \mathbb{R} \backslash\left(-b_{j}, a_{j}\right),
$$

then the Schrödinger-type identity $\operatorname{Sch}_{\alpha}(f g)=\mathrm{fSch}_{\alpha}(g)$ holds.

Proof We first prove

$$
\int_{\mathbb{R}^{n}}(\operatorname{sgn}(x)-\operatorname{sgn}(t)) \hat{f}(x-t) \hat{g_{\varepsilon}}(t) d t=0
$$

from Theorem 2.1.

That is,

$$
\int_{D_{+}}(\operatorname{sgn}(x)-\operatorname{sgn}(t)) \hat{f}(x-t) \hat{g_{\varepsilon}}(t) d t+\int_{D_{-}}(\operatorname{sgn}(x)-\operatorname{sgn}(t)) \hat{f}(x-t) \hat{g_{\varepsilon}}(t) d t=0 .
$$


Let $x \in D_{+}$, if $t \in D_{+}$, the integrand is vanish so (2.1) holds. If $t \in D_{0},(2.1)$ holds since the integration is over a set of measure zero. As for the case $t \in D_{-}$, assume that there exists $t \in D_{-}$, such that $t \in \operatorname{supp} \hat{f}(x-\cdot) \hat{g_{\varepsilon}}(\cdot)$, then $t \in \operatorname{supp} \hat{g_{\varepsilon}} \cap D_{-}, x-t \in \operatorname{supp} \hat{f}$.

Since $D_{-} \cap D_{+}=\emptyset$, there exists $j \in\{1,2, \ldots, n\}$ such that $x_{j} t_{j} \leq 0$. We may assume that $x_{j}>0$ and $t_{j} \leq 0$. Thanks to (2.2), we have $t_{j} \leq-b_{j}$ and $x_{j}-t_{j} \leq b_{j}$, which is impossible.

By repeating this argument for $x \in D_{-}$and $x \in D_{0}$ (see [6]), we find the same conclusion.

Lemma 2.1 Suppose that $f \in L^{p}\left(\mathbb{R}^{n}\right)$ and $g \in L^{q}\left(\mathbb{R}^{n}\right)$, where

$$
\frac{1}{p}+\frac{1}{q}=\frac{1}{r} \leq 1 \quad(1<p, q \leq 2)
$$

Then

$$
(f * g)^{\wedge}=\hat{f} \hat{g_{\varepsilon}}
$$

holds.

Proof Let $y_{i}=1$, where $i=1,2, \ldots, n-1$. Then

$$
f(t, 1, \ldots, 1) \geq k^{b} f(t, k, \ldots, k)
$$

where $k \in(0,1)$.

So

$$
\begin{aligned}
& f\left(t, k^{-1}, \ldots, k^{-1}\right) \geq k^{b} f(t, 1, \ldots, 1), \\
& f\left(t, k y_{1}, \ldots, k y_{n-1}\right) \leq k^{-b} f\left(t, y_{1}, \ldots, y_{n-1}\right), \\
& f(t, k, \ldots, k) \leq k^{-b} f(t, 1, \ldots, 1)
\end{aligned}
$$

where $k \in(0,1)$, which yields

$$
\begin{aligned}
& f_{0^{+}}^{n-2} w(t)>0, \quad f_{0^{+}}^{n-3} w(t)>0, \quad \ldots, \quad f_{0^{+}}^{1} w(t)>0, \quad w(t)>0, \\
& g\left(t, f_{0^{+}}^{n-2} w(t), f_{0^{+}}^{n-3} w(t), \ldots, f_{0^{+}}^{1} w(t), w(t)\right) \\
& \quad \leq g\left(t, f_{0^{+}}^{n-2} A e(t), f_{0^{+}}^{n-3} A e(t), \ldots, f_{0^{+}}^{1} A e(t), A e(t)\right) \\
& \quad \leq g\left(t, f_{0^{+}}^{n-2} A, f_{0^{+}}^{n-3} A, \ldots, f_{0^{+}}^{1} A, A\right) \\
& \quad=g\left(t, \frac{A}{(n-2) !} t^{n-2}, \frac{A}{(n-3) !} t^{n-3}, \ldots, A t, A\right) \\
& \quad \leq g(t, A, A, \ldots, A, A) \\
& \quad \leq A^{b} g(t, 1,1, \ldots, 1,1) \\
& \quad \leq A^{b} g(1,1,1, \ldots, 1,1),
\end{aligned}
$$

and

$$
h\left(t, f_{0^{+}}^{n-2} w(t), f_{0^{+}}^{n-3} w(t), \ldots, f_{0^{+}}^{1} w(t), w(t)\right)
$$




$$
\begin{aligned}
\leq & h\left(t, f_{0^{+}}^{n-2} \frac{1}{A} e(t), f_{0^{+}}^{n-3} \frac{1}{A} e(t), \ldots, f_{0^{+}}^{1} \frac{1}{A} e(t), \frac{1}{A} e(t)\right) \\
= & h\left(t, \frac{\Gamma(\alpha-n+2)}{A \Gamma(\alpha)} t^{\alpha-1}, \frac{\Gamma(\alpha-n+2)}{A \Gamma(\alpha-1)} t^{\alpha-2}, \ldots,\right. \\
& \left.\frac{\Gamma(\alpha-n+2)}{A \Gamma(\alpha-n+3)} t^{\alpha-n+2}, \frac{1}{A} t^{\alpha-n+1}\right) \\
\leq & h\left(t, \frac{\zeta}{A} t^{\alpha-1}, \frac{\zeta}{A} t^{\alpha-1}, \ldots, \frac{\zeta}{A} t^{\alpha-n+3}, \frac{\zeta}{A} t^{\alpha-n+2}\right) \\
\leq & h\left(t, \frac{\zeta}{A} t^{\alpha-1}, \frac{\zeta}{A} t^{\alpha-1}, \ldots, \frac{\zeta}{A} t^{\alpha-n+4}, \frac{\zeta}{A} t^{\alpha-n+3}\right) \\
\leq & \ldots \\
\leq & h\left(t, \frac{\zeta}{A} t^{\alpha-1}, \frac{\zeta}{A} t^{\alpha-1}, \ldots, \frac{\zeta}{A} t^{\alpha-1}, \frac{\zeta}{A} t^{\alpha-1}\right) \\
\leq & \left(\frac{\zeta}{A}\right)^{-b} t^{-b(\alpha-1)} f(t, 1,1, \ldots, 1,1) \\
\leq & \left(\frac{\zeta}{A}\right)^{-b} t^{-b(\alpha-1)} f(0,1,1, \ldots, 1,1) .
\end{aligned}
$$

So

$$
\begin{aligned}
& g\left(t, f_{0^{+}}^{n-2} w(t), f_{0^{+}}^{n-3} w(t), \ldots, f_{0^{+}}^{1} w(t), w(t)\right) \\
& \geq g\left(t, f_{0^{+}}^{n-2} \frac{1}{A} e(t), f_{0^{+}}^{n-3} \frac{1}{A} e(t), \ldots, f_{0^{+}}^{1} \frac{1}{A} e(t), \frac{1}{A} e(t)\right) \\
&= g\left(t, \frac{\Gamma(\alpha-n+2)}{A \Gamma(\alpha)} t^{\alpha-1}, \frac{\Gamma(\alpha-n+2)}{A \Gamma(\alpha-1)} t^{\alpha-2}, \ldots,\right. \\
&\left.\frac{\Gamma(\alpha-n+2)}{A \Gamma(\alpha-n+3)} t^{\alpha-n+2}, \frac{1}{A} t^{\alpha-n+1}\right) \\
& \geq g\left(t, \frac{\zeta}{A} t^{\alpha-1}, \frac{\zeta}{A} t^{\alpha-1}, \ldots, \frac{\zeta}{A} t^{\alpha-n+3}, \frac{\zeta}{A} t^{\alpha-n+2}\right) \\
& \geq g\left(t, \frac{\zeta}{A} t^{\alpha-1}, \frac{\zeta}{A} t^{\alpha-1}, \ldots, \frac{\zeta}{A} t^{\alpha-n+4}, \frac{\zeta}{A} t^{\alpha-n+3}\right) \\
& \geq \ldots \\
& \geq g\left(t, \frac{\zeta}{A} t^{\alpha-1}, \frac{\zeta}{A} t^{\alpha-1}, \ldots, \frac{\zeta}{A} t^{\alpha-1}, \frac{\zeta}{A} t^{\alpha-1}\right) \\
& \geq\left(\frac{\zeta}{A}\right)^{-b} t^{b(\alpha-1)} g(t, 1,1, \ldots, 1,1) \\
& \geq\left(\frac{\zeta}{A}\right)^{-b} t^{b(\alpha-1)} g(0,1,1, \ldots, 1,1)
\end{aligned}
$$

and

$$
\begin{aligned}
& h\left(t, f_{0^{+}}^{n-2} w(t), f_{0^{+}}^{n-3} w(t), \ldots, f_{0^{+}}^{1} w(t), w(t)\right) \\
& \quad \geq h\left(t, f_{0^{+}}^{n-2} A e(t), f_{0^{+}}^{n-3} A e(t), \ldots, f_{0^{+}}^{1} A e(t), A e(t)\right)
\end{aligned}
$$




$$
\begin{aligned}
& \geq h\left(t, f_{0^{+}}^{n-2} A, f_{0^{+}}^{n-3} A, \ldots, f_{0^{+}}^{1} A, A\right) \\
& =h\left(t, \frac{A}{(n-2) !} t^{n-2}, \frac{A}{(n-3) !} t^{n-3}, \ldots, A t, A\right) \\
& \geq f(t, A, A, \ldots, A, A) \\
& \geq A^{-b} f(t, 1,1, \ldots, 1,1) \\
& \geq A^{-b} f(1,1,1, \ldots, 1,1),
\end{aligned}
$$

which yields

$$
\begin{aligned}
& \int_{0}^{1} \int_{0}^{1} H(s, \varsigma) g\left(\varsigma, f_{0^{+}}^{n-2} v(\varsigma), \ldots, f_{0^{+}}^{1} \nu(\varsigma), v(\varsigma)\right) d \varsigma d s \\
& \quad \leq \int_{0}^{1} \iota_{q}\left(\frac{s^{\beta-1}}{\Gamma(\beta)} \int_{0}^{1} g\left(\varsigma, f_{0^{+}}^{n-2} v(\varsigma), \ldots, f_{0^{+}}^{1} \nu(\varsigma), v(\varsigma)\right) d \varsigma\right) d s \\
& \quad \leq \int_{0}^{1} \iota_{q}\left(\frac{s^{\beta-1} A^{b} g(1,1, \ldots, 1)}{\Gamma(\beta)}\right) d s \\
& \quad \leq \int_{0}^{1} \iota_{q}\left(s^{\beta-1}\right) d s
\end{aligned}
$$

and

$$
\begin{aligned}
& \int_{0}^{1} \int_{0}^{1} H(s, \varsigma) f\left(\varsigma, f_{0^{+}}^{n-2} w(\varsigma), \ldots, f_{0^{+}}^{1} w(\varsigma), w(\varsigma)\right) d \varsigma d s \\
& \quad \leq \int_{0}^{1} \iota_{q}\left(\frac{s^{\beta-1}}{\Gamma(\beta)} \int_{0}^{1} f\left(\varsigma, f_{0^{+}}^{n-2} w(\varsigma), \ldots, f_{0^{+}}^{1} w(\varsigma), w(\varsigma)\right) d \varsigma\right) d s \\
& \quad \leq \int_{0}^{1} \iota_{q}\left(\frac{s^{\beta-1}}{\Gamma(\beta)} \int_{0}^{1}\left(\frac{\zeta}{A}\right)^{-b} \varsigma^{-b(\alpha-1)} f(0,1,1, \ldots, 1) d \varsigma\right) d s \\
& \quad \leq \frac{t^{\alpha-n+1}\left(\zeta^{-b} A^{b} f(0,1,1, \ldots, 1)\right)^{q-1}}{\Gamma(\alpha-n+1)(\Gamma(\beta))^{q-1}} \int_{0}^{1} \iota_{q}\left(s^{\beta-1} \int_{0}^{1} \varsigma^{-b(\alpha-1)} d \varsigma\right) d s
\end{aligned}
$$

It follows that

$$
\begin{aligned}
\int_{0}^{1} \iota_{q}\left(\int_{0}^{1} g\left(\varsigma, f_{0^{+}}^{n-2} v(\varsigma), \ldots, f_{0^{+}}^{1} \nu(\varsigma), v(\varsigma)\right) d \varsigma\right) d s \\
\geq \int_{\xi}^{1} \iota_{q}\left(\int_{\xi}^{1} g\left(\varsigma, f_{0^{+}}^{n-2} \nu(\varsigma), \ldots, f_{0^{+}}^{1} \nu(\varsigma), v(\varsigma)\right) d \varsigma\right) d s \\
\geq \int_{\xi}^{1} \gamma(s) \iota_{q}\left(\int_{\xi}^{1} \rho(\varsigma) g\left(\varsigma, f_{0^{+}}^{n-2} v(\varsigma), \ldots, f_{0^{+}}^{1} \nu(\varsigma), v(\varsigma)\right) d \varsigma\right) d s \\
\geq \int_{\xi}^{1} \gamma(s) \iota_{q}\left(\int_{\xi}^{1} \rho(\varsigma)\left(\frac{\zeta}{A}\right)^{b} \varsigma^{b(\alpha-1)} g(0,1,1, \ldots, 1) d \varsigma\right) d s \\
=\left(\zeta^{b} A^{-b} g(0,1,1, \ldots, 1)\right)^{q-1} \int_{\xi}^{1} \gamma(s) \iota_{q}\left(\int_{\xi}^{1} \rho(\varsigma) \varsigma^{b(\alpha-1)} d \varsigma\right) d s \\
\geq t^{\alpha-n+1}\left(\zeta^{b} A^{-b} g(0,1,1, \ldots, 1)\right)^{q-1} \\
\quad \times \int_{\xi}^{1} \gamma(s) \iota_{q}\left(\int_{\xi}^{1} \rho(\varsigma) \varsigma^{b(\alpha-1)} d \varsigma\right) d s
\end{aligned}
$$


and

$$
\begin{aligned}
& \int_{0}^{1} \int_{0}^{1} h\left(\varsigma, f_{0^{+}}^{n-2} w(\varsigma), \ldots, f_{0^{+}}^{1} w(\varsigma), w(\varsigma)\right) d \varsigma d s \\
& \quad \geq \int_{\xi}^{1} \iota_{q}\left(\int_{\xi}^{1} f\left(\varsigma, f_{0^{+}}^{n-2} w(\varsigma), \ldots, f_{0^{+}}^{1} w(\varsigma), w(\varsigma)\right) d \varsigma\right) d s \\
& \quad \geq \int_{\xi}^{1} \gamma(s) \iota_{q}\left(\int_{\xi}^{1} \rho(\varsigma) f\left(\varsigma, f_{0^{+}}^{n-2} w(\varsigma), \ldots, f_{0^{+}}^{1} w(\varsigma), w(\varsigma)\right) d \varsigma\right) d s \\
& \quad \geq \int_{\xi}^{1} \gamma(s) \iota_{q}\left(\int_{\xi}^{1} \rho(\varsigma) H(\varsigma, \varsigma) A^{-b} f(1,1,1, \ldots, 1) d \varsigma\right) d s \\
& \quad \geq\left(A^{-b} f(1,1,1, \ldots, 1)\right)^{q-1} \int_{\xi}^{1} \gamma(s) \iota_{q}\left(\int_{\xi}^{1} \rho(\varsigma) H(\varsigma, \varsigma) d \varsigma\right) d s \\
& \quad \geq t^{\alpha-n+1}\left(A^{-b} f(1,1,1, \ldots, 1)\right)^{q-1} \int_{\xi}^{1} \gamma(s) \iota_{q}\left(\int_{\xi}^{1} \rho(\varsigma) H(\varsigma, \varsigma) d \varsigma\right) d s,
\end{aligned}
$$

which yields

$$
T(v, w)(t) \geq \frac{1}{A} t^{\alpha-n+1}=\frac{1}{A} e(t),
$$

where $t \in(0,1)$.

Then we prove that $T: Q_{e} \times Q_{e} \rightarrow Q_{e}$ is a mixed monotone operator. We have

$$
\begin{aligned}
& \int_{0}^{1} \iota_{q}\left(\int_{0}^{1} g\left(\varsigma, f_{0^{+}}^{n-2} v_{1}(\varsigma), \ldots, f_{0^{+}}^{1} \nu_{1}(\varsigma), v_{1}(\varsigma)\right) d \varsigma\right) d s \\
& \quad \leq \int_{0}^{1} \iota_{q}\left(\int_{0}^{1} H(s, \varsigma) g\left(\varsigma, f_{0^{+}}^{n-2} v_{2}(\varsigma), \ldots, f_{0^{+}}^{1} \nu_{2}(\varsigma), v_{2}(\varsigma)\right) d \varsigma\right) d s .
\end{aligned}
$$

Thus $T(v, w)(t)$ is nondecreasing in $v$ for any $w \in Q_{e}$.

Let $w_{1}, w_{2} \in Q_{e}$ and $w_{1} \geq w_{2}$. Then

$$
\begin{aligned}
& \int_{0}^{1} P(s, \varsigma) \iota_{q}\left(\int_{0}^{1} Q(s, \varsigma) f\left(\varsigma, f_{0^{+}}^{n-2} w_{1}(\varsigma), \ldots, f_{0^{+}}^{1} w_{1}(\varsigma), w_{1}(\varsigma)\right) d \varsigma\right) d s \\
& \quad \leq \int_{0}^{1} P(s, \varsigma) \iota_{q}\left(\int_{0}^{1} Q(s, \varsigma) f\left(\varsigma, f_{0^{+}}^{n-2} w_{2}(\varsigma), \ldots, f_{0^{+}}^{1} w_{2}(\varsigma), w_{2}(\varsigma)\right) d \varsigma\right) d s
\end{aligned}
$$

i.e.,

$$
T\left(v, w_{1}\right)(t) \leq T\left(v, w_{2}\right)(t), \quad w \in Q_{e}
$$

Therefore $T(v, w)(t)$ is nonincreasing in $w$ for any $v \in Q_{e}$.

We shall show that the operator $T$ has a fixed point.

It follows that

$$
\begin{aligned}
& \int_{0}^{1} P(s, \varsigma) \iota_{q}\left(\int_{0}^{1} Q(s, \varsigma) g\left(\varsigma, f_{0^{+}}^{n-2} t v(\varsigma), \ldots, f_{0^{+}}^{1} t v(\varsigma), t v(\varsigma)\right) d \varsigma\right) d s \\
& \quad=\int_{0}^{1} P(s, \varsigma) \iota_{q}\left(\int_{0}^{1} Q(s, \varsigma) g\left(\varsigma, t f_{0^{+}}^{n-2} v(\varsigma), \ldots, t f_{0^{+}}^{1} \nu(\varsigma), t v(\varsigma)\right) d \varsigma\right) d s
\end{aligned}
$$




$$
\begin{aligned}
& \geq \int_{0}^{1} P(s, \varsigma) \iota_{q}\left(\int_{0}^{1} Q(s, \varsigma) t^{b} g\left(\varsigma, f_{0^{+}}^{n-2} v(\varsigma), \ldots, f_{0^{+}}^{1} \nu(\varsigma), \nu(\varsigma)\right) d \varsigma\right) d s \\
& \geq t^{b} \int_{0}^{1} P(s, \varsigma) \iota_{q}\left(\int_{0}^{1} Q(s, \varsigma) g\left(\varsigma, f_{0^{+}}^{n-2} v(\varsigma), \ldots, f_{0^{+}}^{1} \nu(\varsigma), \nu(\varsigma)\right) d \varsigma\right) d s
\end{aligned}
$$

and

$$
\begin{aligned}
& \int_{0}^{1} P(s, \varsigma) \iota_{q}\left(\int_{0}^{1} Q(s, \varsigma) f\left(\varsigma, f_{0^{+}}^{n-2} t^{-1} w(\varsigma), \ldots, f_{0^{+}}^{1} t^{-1} w(\varsigma), t^{-1} w(\varsigma)\right) d \varsigma\right) d s \\
& \quad=\int_{0}^{1} P(s, \varsigma) \iota_{q}\left(\int_{0}^{1} Q(s, \varsigma) f\left(\varsigma, t^{-1} f_{0^{+}}^{n-2} w(\varsigma), \ldots, t^{-1} f_{0^{+}}^{1} w(\varsigma), t^{-1} w(\varsigma)\right) d \varsigma\right) d s \\
& \quad \geq \int_{0}^{1} P(s, \varsigma) \iota_{q}\left(\int_{0}^{1} Q(s, \varsigma) t^{b} f\left(\varsigma, f_{0^{+}}^{n-2} w(\varsigma), \ldots, f_{0^{+}}^{1} w(\varsigma), w(\varsigma)\right) d \varsigma\right) d s \\
& \quad \geq t^{b} \int_{0}^{1} P(s, \varsigma) \iota_{q}\left(\int_{0}^{1} Q(s, \varsigma) f\left(\varsigma, f_{0^{+}}^{n-2} w(\varsigma), \ldots, f_{0^{+}}^{1} w(\varsigma), w(\varsigma)\right) d \varsigma\right) d s,
\end{aligned}
$$

we obtain

$$
T\left(t x, \frac{1}{t} y\right) \geq t^{b} T(x, y), \quad x, y \in Q_{e}, t \in(0,1), b \in(0,1) .
$$

Therefore

$$
\begin{aligned}
\frac{\Gamma(\alpha-n+2)}{A \Gamma(\alpha)} t^{\alpha-1} & =\frac{1}{A} f_{0^{+}}^{n-2} e(t) \leq u(t) \\
& \leq M f_{0^{+}}^{n-2} e(t)=\frac{A \Gamma(\alpha-n+2)}{\Gamma(\alpha)} t^{\alpha-1}, \quad t \in(0,1) .
\end{aligned}
$$

Since $f \in L^{p}\left(\mathbb{R}^{n}\right)$ and $g \in L^{q}\left(\mathbb{R}^{n}\right)$ (see [11]), we have

$$
f * g \in L^{r}\left(\mathbb{R}^{n}\right)
$$

which shows that there exist functions $g_{n} \in \mathcal{S}\left(\mathbb{R}^{n}\right)$ such that

$$
\begin{gathered}
\left\|g-g_{n}\right\|_{q} \rightarrow 0 \\
\text { as } n \rightarrow \infty, \\
\left(f * g_{n}\right)^{\wedge}=\hat{f} \hat{g}_{\varepsilon_{n}},
\end{gathered}
$$

and

$$
\left(f g_{n}\right)^{\wedge}=\hat{f} * \hat{g}_{\varepsilon_{n}} .
$$

Thus in the distributional sense

$$
\lim _{n \rightarrow \infty}\left(f * g_{n}\right)^{\wedge}(x)=(f * g)^{\wedge}(x) .
$$


On the other hand

$$
\left|\left\langle\hat{f}\left(\hat{g_{\varepsilon_{n}}}-\hat{g_{\varepsilon}}, \lambda\right) \mid\right\rangle=\right|\left\langle\left(\hat{g_{\varepsilon_{n}}}-\hat{g_{\varepsilon}}, \hat{f} \lambda\right) \mid\right\rangle \leq\|\hat{f} \lambda\|_{q}\left\|\hat{g_{\varepsilon_{n}}}-\hat{g_{\varepsilon}}\right\|_{q^{\prime}} \rightarrow 0
$$

as $n \rightarrow \infty$.

Hence the result

$$
(f * g)^{\wedge}=\hat{f} \hat{g_{\varepsilon}}
$$

is obtained.

We define

$$
\begin{aligned}
& S_{n}=\left\{\sigma_{k}:\{1,2, \ldots, n\} \rightarrow\{+1,-1\}\right\}, \\
& Q_{\sigma_{k}}=\left\{y=\left(y_{1}, y_{2}, \ldots, y_{n}\right) \in \mathbb{R}^{n}: y_{j} \sigma_{k}(j)>0\right\}
\end{aligned}
$$

and

$$
-Q_{\sigma_{k}}=\left\{\xi \in \mathbb{R}^{n}:-\xi \in Q_{\sigma_{k}}\right\}, \quad \operatorname{sgn}(\xi)=\prod_{j=1}^{n} \operatorname{sgn}\left(\xi_{j}\right),
$$

where $j=1,2, \ldots, n$.

It follows that if $\xi \in Q_{\sigma_{k}}$ and $\eta \in-Q_{\sigma_{k}}$, then $\operatorname{sgn}(\xi)=\operatorname{sgn}(\eta)$ when $n$ is an even, and $\operatorname{sgn}(\xi)=-\operatorname{sgn}(\eta)$ when $n$ is an odd.

With these notations we have the following.

Theorem 2.2 Let $n$ be an odd and $f \in \mathcal{S}\left(\mathbb{R}^{n}\right), g \in L^{p}\left(\mathbb{R}^{n}\right)(1<p \leq 2)$ satisfy supp $\hat{f}$, $\operatorname{supp} \hat{g_{\varepsilon}} \subseteq Q_{\sigma_{k}} \cup-Q_{\sigma_{k}}$ with $a_{j} \sigma_{k}(j),-b_{j} \sigma_{k}(j) \in \operatorname{supp} \hat{f}(j=1,2, \ldots, n)$ and

$$
\operatorname{supp} \hat{f} \subseteq\left\{\xi \in Q_{\sigma_{k}}: \sigma_{k}(j) \xi_{j} \leq a_{j}\right\} \cup\left\{\xi \in-Q_{\sigma_{k}}:-\sigma_{k}(j) \xi_{j} \leq b_{j}\right\}
$$

Then $g \in L^{p}\left(\mathbb{R}^{n}\right)$ satisfies the Schrödinger-type identity $\operatorname{Sch}_{\alpha}(f g)=\operatorname{fSch}_{\alpha}(g)$ if and only if

$$
\operatorname{supp} \hat{f} \subseteq\left\{\xi \in Q_{\sigma_{k}}: \sum_{j=1}^{n} \frac{\sigma_{k}(j) \xi_{j}}{b_{j}} \geq 1\right\} \cup\left\{\xi \in \sum_{j=1}^{n} \frac{-\sigma_{k}(j) \xi_{j}}{a_{j}} \geq 1\right\} .
$$

Proof Suppose that $Q_{\sigma_{k}}$ is the first octant in $\mathbb{R}^{n}$, that is to say, all the $\sigma_{k}(j)=1$, where $j=1,2, \ldots, n$.

Let

$$
\begin{aligned}
\hat{f}\left(s, g_{0^{+}}^{n-2} \operatorname{sgn}(s), g_{0^{+}}^{n-3} \operatorname{sgn}(s), \ldots, g_{0^{+}}^{1} \operatorname{sgn}(s), \operatorname{sgn}(s)\right) \\
\quad= \begin{cases}f\left(s, g_{0^{+}}^{n-2} m(s), g_{0^{+}}^{n-3} m(s), \ldots, g_{0^{+}}^{1} m(s), m(s)\right), & \operatorname{sgn}(s)<m(s), \\
f\left(s, g_{0^{+}}^{n-2} \operatorname{sgn}(s), g_{0^{+}}^{n-3} \operatorname{sgn}(s), \ldots, g_{0^{+}}^{1} \operatorname{sgn}(s), \operatorname{sgn}(s)\right), & m(s) \leq \operatorname{sgn}(s) \leq n(s), \\
f\left(s, g_{0^{+}}^{n-2} n(s), g_{0^{+}}^{n-3} n(s), \ldots, g_{0^{+}}^{1} n(s), n(s)\right), & \operatorname{sgn}(s)>n(s) .\end{cases}
\end{aligned}
$$


Consider the fractional differential equation

$$
\begin{aligned}
& Q_{0^{+}}^{\beta} \iota_{p}\left(Q_{0^{+}}^{\alpha-n+2} n(s)\right)+\hat{f}\left(s, g_{0^{+}}^{n-2} \operatorname{sgn}(s), g_{0^{+}}^{n-3} \operatorname{sgn}(s), \ldots, g_{0^{+}}^{1} \operatorname{sgn}(s), \operatorname{sgn}(s)\right)=0, \\
& 0<t<1, \\
& v(0)=0, \quad v(1)=a v(\xi), \quad Q_{0^{+}}^{\alpha-n+2} v(0)=Q_{0^{+}}^{\alpha-n+2} v(1)=0 .
\end{aligned}
$$

Set $\Omega_{2}=\left\{v \in E_{2}:\|v\| \leq M_{1} \iota_{q}\left(M_{2} L_{2}\right)\right\}$, then $\Omega_{2}$ is a closed, bounded and convex set, where

$$
L_{2}:=\sup _{t \in[0,1], v \in \Omega_{2}}\left|\hat{f}\left(s, g_{0^{+}}^{n-2} \operatorname{sgn}(s), \ldots, g_{0^{+}}^{1} \operatorname{sgn}(s), \operatorname{sgn}(s)\right)\right|+1 .
$$

The operator $A: \Omega_{2} \rightarrow E_{2}$ is defined by

$$
A \operatorname{sgn}(s)=\int_{0}^{1} P(s, \varsigma) \iota_{q}\left(\int_{0}^{1} Q(s, \varsigma) \hat{f}\left(\varsigma, g_{0^{+}}^{n-2} \nu(\varsigma), \ldots, g_{0^{+}}^{1} \nu(\varsigma), \nu(\varsigma)\right) d \varsigma\right) d s .
$$

Now, we show that $A$ is a completely continuous operator. It follows that

$$
\begin{aligned}
& |(A v)(s)| \\
& \quad=\left|\int_{0}^{1} P(s, \varsigma) \iota_{q}\left(\int_{0}^{1} Q(s, \varsigma) \hat{f}\left(\varsigma, g_{0^{+}}^{n-2} v(\varsigma), \ldots, g_{0^{+}}^{1} \nu(\varsigma), v(\varsigma)\right) d \varsigma\right) d s\right| \\
& \quad \leq \int_{0}^{1} P(s, \varsigma) \iota_{q}\left(\int_{0}^{1} Q(s, \varsigma)\left|\hat{f}\left(\varsigma, g_{0^{+}}^{n-2} v(\varsigma), \ldots, g_{0^{+}}^{1} \nu(\varsigma), v(\varsigma)\right)\right| d \varsigma\right) d s \\
& \quad \leq L_{2}^{q-1} \int_{0}^{1} P(s, \varsigma) \iota_{q}\left(\int_{0}^{1} Q(s, \varsigma) d \varsigma\right) d s \\
& \quad \leq L_{2}^{q-1} \int_{0}^{1} P(s, s) \iota_{q}\left(\int_{0}^{1} Q(\varsigma, \varsigma) d \varsigma\right) d s \\
& \quad<+\infty
\end{aligned}
$$

which yields

$$
P\left(t_{1}, s\right)-P\left(t_{2}, s\right)<\frac{\varepsilon}{L_{2}^{q-1} \iota_{q}\left(\int_{0}^{1} Q(\varsigma, \varsigma) d \varsigma\right)} .
$$

So

$$
\begin{aligned}
& \left|A v\left(t_{2}\right)-A v\left(t_{1}\right)\right| \\
& \quad \leq \int_{0}^{1}\left|P\left(t_{2}, s\right)-P\left(t_{1}, s\right)\right| \iota_{q}\left(\int_{0}^{1} Q(s, \varsigma) \hat{f}\left(\varsigma, g_{0^{+}}^{n-2} v(\varsigma), \ldots, g_{0^{+}}^{1} \nu(\varsigma), v(\varsigma)\right) d \varsigma\right) d s \\
& \quad \leq L_{2}^{q-1} \int_{0}^{1}\left|P\left(t_{2}, s\right)-P\left(t_{1}, s\right)\right| \iota_{q}\left(\int_{0}^{1} Q(\varsigma, \varsigma) d \varsigma\right) d s \\
& \quad \leq L_{2}^{q-1} \iota_{q}\left(\int_{0}^{1} Q(\varsigma, \varsigma) d \varsigma\right) \int_{0}^{1}\left|P\left(t_{2}, s\right)-P\left(t_{1}, s\right)\right| d s \\
& \quad<\varepsilon
\end{aligned}
$$

for any $v \in \Omega_{2}$. 
We prove that the fractional differential equation has at least one positive solution. Suppose that $d(s)$ is a solution of (2.4) (see [9]), then

$$
d(0)=0, \quad d(1)=a d(\xi), \quad Q_{0^{+}}^{\alpha-n+2} d(0)=Q_{0^{+}}^{\alpha-n+2} d(1)=0 .
$$

So

$$
\begin{aligned}
& f\left(s, g_{0^{+}}^{n-2} n(s), g_{0^{+}}^{n-3} n(s), \ldots, g_{0^{+}}^{1} n(s), n(s)\right) \\
& \quad \leq \hat{f}\left(s, g_{0^{+}}^{n-2} d(s), g_{0^{+}}^{n-3} d(s), \ldots, g_{0^{+}}^{1} d(s), d(s)\right) \\
& \quad \leq f\left(s, g_{0^{+}}^{n-2} m(s), g_{0^{+}}^{n-3} m(s), \ldots, g_{0^{+}}^{1} m(s), m(s)\right) .
\end{aligned}
$$

So

$$
\begin{aligned}
f(s, & \left.g_{0^{+}}^{n-2} q(s), g_{0^{+}}^{n-3} q(s), \ldots, g_{0^{+}}^{1} q(s), q(s)\right) \\
& \leq \hat{f}\left(s, g_{0^{+}}^{n-2} d(s), g_{0^{+}}^{n-3} d(s), \ldots, g_{0^{+}}^{1} d(s), d(s)\right) \\
& \leq f\left(s, g_{0^{+}}^{n-2} p(s), g_{0^{+}}^{n-3} p(s), \ldots, g_{0^{+}}^{1} p(s), p(s)\right),
\end{aligned}
$$

which yields

$$
\begin{aligned}
Q_{0^{+}}^{\beta} \iota_{p}\left(Q_{0^{+}}^{\alpha-n+2} n(s)\right) & =Q_{0^{+} \iota_{p}}^{\beta}\left(Q_{0^{+}}^{\alpha-n+2}(F p)(s)\right) \\
& =f\left(s, g_{0^{+}}^{n-2} p(s), g_{0^{+}}^{n-3} p(s), \ldots, g_{0^{+}}^{1} p(s), p(s)\right) .
\end{aligned}
$$

From the above discussions, we have

$$
\begin{aligned}
& Q_{0^{+}}^{\beta} \iota_{p}\left(Q_{0^{+}}^{\alpha-n+2} n(s)\right)-Q_{0^{+}}^{\beta} \iota_{p}\left(Q_{0^{+}}^{\alpha-n+2} d(s)\right) \\
& =f\left(s, g_{0^{+}}^{n-2} p(s), g_{0^{+}}^{n-3} p(s), \ldots, g_{0^{+}}^{1} p(s), p(s)\right) \\
& \quad-\hat{f}\left(s, g_{0^{+}}^{n-2} d(s), g_{0^{+}}^{n-3} d(s), \ldots, g_{0^{+}}^{1} d(s), d(s)\right) \\
& \quad \geq 0,
\end{aligned}
$$

where $t \in[0,1]$.

If we let $z(s)=\iota_{p}\left(Q_{0^{+}}^{\alpha-n+2} n(s)\right)-\iota_{p}\left(Q_{0^{+}}^{\alpha-n+2} d(s)\right)$, then $z(0)=z(1)=0$. By Lemma 2.1, we have $z(s) \leq 0$.

Hence,

$$
\iota_{p}\left(Q_{0^{+}}^{\alpha-n+2} n(s)\right) \leq \iota_{p}\left(Q_{0^{+}}^{\alpha-n+2} d(s)\right)
$$

where $s \in[0,1]$.

Since $\iota_{p}$ is monotone increasing,

$$
Q_{0^{+}}^{\alpha-n+2} n(s) \leq Q_{0^{+}}^{\alpha-n+2} d(s),
$$

that is,

$$
Q_{0^{+}}^{\alpha-n+2}(n-d)(s) \leq 0 .
$$


By the assumption that $\operatorname{supp} \hat{f}$, supp $\hat{g_{\varepsilon}} \subseteq \sigma_{k}(j) \cup-\sigma_{k}(j)$, we obtain

$$
\int_{Q_{\sigma_{k}}} \hat{f}(x-t) \hat{g_{\varepsilon}}(s) d s=0
$$

where $x \in-Q_{\sigma_{k}}$, and

$$
\int_{-Q_{\sigma_{k}}} \hat{f}(x-t) \hat{g_{\varepsilon}}(s) d s=0
$$

where $x \in Q_{\sigma_{k}}$.

So

$$
\operatorname{supp} \hat{g_{\varepsilon}} \chi Q_{\sigma_{k}} \subseteq\left\{\xi \in Q_{\sigma_{k}}: \sum_{j=1}^{n} \frac{\xi_{j}}{b_{j}} \geq 1\right\}
$$

as the other case can be obtained in a similar way.

Let $\lambda=\hat{f} \chi_{-Q_{\sigma_{k}}}$ and $\varrho=\hat{g_{\varepsilon}} \chi Q_{\sigma_{k}}$. We decompose $\varrho$ into

$$
\varrho=\varrho_{1}+\varrho_{2}
$$

with supp $\varrho_{1} \subseteq \prod_{j=1}^{n}\left[0, b_{j}\right]$ and $\operatorname{supp} \varrho_{2} \subseteq \overline{Q_{\sigma_{k}} \backslash \prod_{j=1}^{n}\left(0, b_{j}\right)}$.

By (2.5) we obtain

$$
\left(\varrho_{1} * \lambda\right)(x)=-\left(\varrho_{2} * \lambda\right)(x)
$$

where $x \in-Q_{\sigma_{k}}$.

Meanwhile

$$
\operatorname{supp}\left(\varrho_{2} * \lambda\right) \subseteq \operatorname{supp} \varrho_{2}+\operatorname{supp} \lambda \subseteq \overline{Q_{\sigma_{k}} \backslash \prod_{j=1}^{n}\left(0, b_{j}\right)}+\prod_{j=1}^{n}\left[-b_{j}, 0\right] \subseteq \mathbb{R}^{n} \backslash\left(-Q_{\sigma_{k}}\right)
$$

and

$$
\operatorname{supp}\left(\varrho_{1} * \lambda\right) \subseteq \operatorname{supp} \varrho_{1}+\operatorname{supp} \lambda \subseteq \prod_{j=1}^{n}\left[0, b_{j}\right]+\prod_{j=1}^{n}\left[-b_{j}, 0\right] \subseteq \prod_{j=1}^{n}\left[-b_{j}, b_{j}\right]
$$

By (2.6) it is clear that

$$
\operatorname{supp}\left(\varrho_{1} * \lambda\right) \subseteq \mathbb{R}^{n} \backslash(-) Q_{\sigma_{k}} .
$$

This together with (2.7) implies that

$$
\operatorname{conv} \operatorname{supp}\left(\varrho_{1} * \lambda\right) \subseteq\left\{\xi \in \mathbb{R}^{n}:-b_{j} \leq \xi_{j} \leq b_{j}, \sum_{j=1}^{n} \frac{\xi_{j}}{a_{j}} \geq 1-n\right\}
$$


We claim that, for any $\xi \in \operatorname{supp} \varrho_{1}$,

$$
\sum_{j=1}^{n} \frac{\xi_{j}}{a_{j}} \geq 1
$$

holds.

If it is invalid, then there is $\xi^{1} \in \operatorname{conv} \operatorname{supp} \varrho_{1}$ satisfying

$$
\sum_{j=1}^{n} \frac{\xi_{j}}{b_{j}}<1
$$

Note that $\xi^{2}=b \in \operatorname{supp} \lambda$ satisfies

$$
\sum_{j=1}^{n} \frac{\xi_{j}}{b_{j}}=-n
$$

Since

$$
\operatorname{conv} \operatorname{supp}\left(\varrho_{1} * \lambda\right)=\operatorname{conv} \operatorname{supp} \varrho_{1}+\operatorname{conv} \operatorname{supp} \lambda,
$$

there exists some point $\xi \in \operatorname{conv} \operatorname{supp}\left(\varrho_{1} * \lambda\right)$ such that

$$
\sum_{j=1}^{n} \frac{\xi_{j}}{a_{j}}<1-n
$$

This contradicts (2.8). We conclude that

$$
\operatorname{supp} \varrho_{1}=\operatorname{supp} \hat{g_{\varepsilon}} \chi Q_{\sigma_{k}} \subseteq\left\{\xi \in Q_{\sigma_{k}}: \sum_{j=1}^{n} \frac{\xi_{j}}{b_{j}} \geq 1\right\} .
$$

This completes the proof.

\section{Conclusions}

This paper was mainly devoted to developing the Schrödinger-type identity for a Schrödinger free boundary problem in $\mathbb{R}^{n}$. As an application, we established necessary and sufficient conditions for the product of some distributional functions to satisfy the Schrödinger-type identity. As a consequence, our results significantly improved and generalized previous work.

Acknowledgements

The authors are thankful to the honorable reviewers for their valuable suggestions and comments, which improved the paper.

Funding

Not applicable. 
Availability of data and materials

Not applicable.

Ethics approval and consent to participate

Not applicable.

Competing interests

The authors declare that they have no competing interests.

Consent for publication

Not applicable.

Authors' contributions

All authors read and approved the final manuscript.

\section{Publisher's Note}

Springer Nature remains neutral with regard to jurisdictional claims in published maps and institutional affiliations.

Received: 17 July 2018 Accepted: 4 September 2018 Published online: 11 September 2018

\section{References}

1. Aubin, J.P., Ekeland, I.: Applied Nonlinear Analysis. Wiley, New York (1984)

2. Bedrosian, E.: A product theorem for Hilbert transform. Proc. IEEE 51, 868-869 (1963)

3. Brown, J.: Analytic signals and product theorems for Hilbert transforms. IEEE Trans. Circuits Syst. 21, 790-792 (1974)

4. Brown, J.: A Hilbert transform product theorem. Proc. IEEE 74, 520-521 (1986)

5. Chen, X., Zhang, L.: New results on the existences of solutions of the Dirichlet problem with respect to the Schrödinger-prey operator and their applications. J. Inequal. Appl. 2017, 143 (2017)

6. Cohen, L.: Time-Frequency Analysis: Theory and Applications. Prentice Hall, Englewood Cliffs (1995)

7. Donoghue, W.: Distributions and Fourier Transforms. Academic Press, New York (1969)

8. Gabor, D.: Theory of communication. Proc. Inst. Electr. Eng. 93, 429-457 (1946)

9. Gasquet, C., Witomski, P.: Fourier Analysis and Applications. Springer, New York (1999)

10. Huang, J.: A new type of minimal thinness with respect to the stationary Schrödinger operator and its applications. Monatshefte Math. 183(3), 495-507 (2017)

11. Kokilashvili, V.: Singular operators in weighted spaces. In: Colloquia Mathematica Societatis Janos Bolyai, vol. 35, Functions, Series, Operators, Budapest, Hungary (1980)

12. Laskin, N.: Fractional quantum mechanics and Lévy path integrals. Phys. Lett. A 268, 298-305 (2000)

13. Laskin, N.: Fractional Schrödinger equation. Phys. Rev. E 66, 056108 (2002)

14. LV, F., Ulker, T.: Existences of weak solutions for two point boundary value problems of Schrödingerean predator-prey system ant their applications. Adv. Differ. Equ. 2017, 159 (2017)

15. Nuttall, A., Bedrosian, E.: On the quadrature approximation for the Hilbert transform of modulated signals. Proc. IEEE 54, 1458-1459 (1966)

16. Oppenheim, A., Lim, J.: The importance of phase in signal. Proc. IEEE 69, 529-541 (1981)

17. Pandey, J.: The Hilbert Transform of Schwartz Distributions and Applications. Wiley, New York (1996)

18. Papageorgiou, N.S., Kyritsi-Yiallourou, S.Th.: Handbook of Applied Analysis. Advance in Mechanics and Mathematics, vol. 19. Springer, New York (2009)

19. Wan, L.: Some remarks on Phragmén-Lindelöf theorems for weak solutions of the stationary Schrödinger operator. Bound. Value Probl. 2015, 239 (2015)

20. Yang, D., Ren, Y.: Dirichlet problem on the upper half space. Proc. Indian Acad. Sci. Math. Sci. 124(2), 175-178 (2014)

\section{Submit your manuscript to a SpringerOpen ${ }^{\circ}$ journal and benefit from:}

- Convenient online submission

- Rigorous peer review

- Open access: articles freely available online

- High visibility within the field

- Retaining the copyright to your article

Submit your next manuscript at $\boldsymbol{~ s p r i n g e r o p e n . c o m ~}$ 